\title{
Enhancement of Kondo Effect in Quantum Dots with an Even Number of Electrons
}

\author{
Mikio Eto ${ }^{1,2}$ and Yuli V. Nazarov ${ }^{1}$ \\ ${ }^{1}$ Department of Applied Physics/DIMES, Delft University of Technology, Lorentzweg 1, 2628 CJ Delft, The Netherlands \\ ${ }^{2}$ Faculty of Science and Technology, Keio University, 3-14-1 Hiyoshi, Kohoku-ku, Yokohama 223-8522, Japan
}

(Received 31 January 2000)

\begin{abstract}
We investigate the Kondo effect in a quantum dot with almost degenerate spin-singlet and triplet states for an even number of electrons. We show that the Kondo temperature as a function of the energy difference between the states $\Delta$ reaches its maximum around $\Delta=0$ and decreases with increasing $\Delta$. The Kondo effect is thus enhanced by competition between singlet and triplet states. Our results explain recent experimental findings. We evaluate the linear conductance in the perturbative regime.
\end{abstract}

PACS numbers: 73.23.Hk, 72.15.Qm, 85.30.Vw

The Kondo effect [1] takes place when a localized spin $S$ is brought in contact with the electron Fermi sea. The Kondo effect gives rise to a new many-body ground state that has a lesser spin. Recently the Kondo effect has been observed in semiconductor quantum dots connected to external leads by tunnel junctions [2]. In this case the localized spin is formed by electrons in the dot. The number of electrons $N$ is fixed by the Coulomb blockade to integer values and can be tuned by gate voltage. Despite the Coulomb blockade, the ground state of the dot is usually similar to what one obtains disregarding the interaction. The discrete spin-degenerate levels in the dot are consecutively occupied, and the total spin is zero or $1 / 2$ for an even and odd number of electrons, respectively. Then the Kondo effect takes place only in the latter case $[3,4]$.

Significant deviations from this plain picture were recently observed in so-called "vertical" quantum dots $[5,6]$. The strength of the electron-electron Coulomb interaction in such dots is comparable with the spacing of discrete levels, and this may give rise to a complicated ground state. For example, if two electrons are put into nearly degenerate levels, the exchange interaction favors a spin-triplet state. This state can be changed to a spin singlet by applying a magnetic field since the magnetic field increases the level spacing [5].

This gives a unique possibility to change the spin of the ground state during an experiment and even obtain extra degenerate states by tuning the energies of different spin configurations to the same value. Such a possibility hardly exists in the traditional solid state context. The Kondo effect in multilevel quantum dots has been investigated by several groups $[7,8]$. In this Letter we examine a novel effect that stems from the competition between spin-singlet and threefold spin-triplet states for an even number of electrons in a dot. Since the energy difference between the states $\Delta$ can be controlled experimentally, we elucidate $\Delta$ dependence of Kondo temperature as a typical energy scale for the Kondo effect and linear conductance through the dot. This enables direct comparison between our calculations and recent experimental results [9].

At large positive $\Delta$ the dot is in a triplet ground state and an extra singlet state can be disregarded. The Kondo effect follows a usual $S=1$ scenario. At large negative $\Delta$ the ground state of the dot is a spin singlet and the Kondo effect ceases to exist. From this one could suggest that the Kondo temperature decreases as $\Delta \rightarrow 0$ from the positive side. Our results show just the opposite. The Kondo temperature $T_{K}(\Delta)$ is enhanced at small $\Delta$ and reaches its maximum at $\Delta \approx T_{K}^{\max }$. At $\Delta \gg T_{K}^{\max }$ the Kondo temperature decreases with increasing $\Delta$ obeying a power law $T_{K}(\Delta) \propto 1 / \Delta^{\gamma}$. The exponent $\gamma$ is not universal but depends on model parameters. Our results clearly demonstrate the importance of one of the basic principles of Kondo physics: although the Kondo effect occurs at small energy scale $T_{K}$, the value of this scale is determined by all energies from $T_{K}$ up to the upper cutoff. In our case, the energies from $\Delta$ to the upper cutoff would feel fourfold degeneracy of the dot states, which enhances the Kondo temperature. At $\Delta<\Delta_{c}\left(\left|\Delta_{c}\right| \sim T_{K}^{\max }\right)$, the Kondo effect is not relevant. We stress the difference between this mechanism and the one proposed in Ref. [8], where the Kondo effect arises from extra degeneracy between one component of the spin-triplet state and a singlet state, which is brought by the Zeeman splitting.

To model the situation, it is sufficient to consider two extra electrons in a quantum dot at the background of a singlet state of all other $N-2$ electrons, which we will regard as the vacuum. These two extra electrons occupy two levels of different orbital symmetry [10]. The energies of the levels are $\varepsilon_{1}, \varepsilon_{2}$. Possible two-electron states are (i) the threefold spin-triplet state, (ii) the spin-singlet state of the same orbital symmetry as the triplet state, $1 / \sqrt{2}\left(d_{1 \uparrow}^{\dagger} d_{2 \downarrow}^{\dagger}-d_{1 \downarrow}^{\dagger} d_{2 \uparrow}^{\dagger}\right)|0\rangle$, and (iii) two singlets of different orbital symmetry, $d_{1 \uparrow}^{\dagger} d_{1 \downarrow}^{\dagger}|0\rangle, d_{2 \uparrow}^{\dagger} d_{2 \downarrow}^{\dagger}|0\rangle$. Among the singlet states, we consider only a state of the lowest energy, which belongs to the group (iii). Thus we restrict our attention to four states, $|S M\rangle$ :

$$
\begin{gathered}
|11\rangle=d_{1 \uparrow}^{\dagger} d_{2 \uparrow}^{\dagger}|0\rangle, \\
|10\rangle=\frac{1}{\sqrt{2}}\left(d_{1 \uparrow}^{\dagger} d_{2 \downarrow}^{\dagger}+d_{1 \downarrow}^{\dagger} d_{2 \uparrow}^{\dagger}\right)|0\rangle, \\
|1-1\rangle=d_{1 \downarrow}^{\dagger} d_{2 \downarrow}^{\dagger}|0\rangle,
\end{gathered}
$$




$$
|00\rangle=\frac{1}{\sqrt{2}}\left(C_{1} d_{1 \uparrow}^{\dagger} d_{1 \downarrow}^{\dagger}-C_{2} d_{2 \uparrow}^{\dagger} d_{2 \downarrow}^{\dagger}\right)|0\rangle,
$$

where $d_{i \sigma}^{\dagger}$ creates an electron with spin $\sigma$ in level $i$. Their energies, $E_{S=1}, E_{S=0}$, and coefficients in the singlet state, $C_{1}, C_{2}\left(\left|C_{1}\right|^{2}+\left|C_{2}\right|^{2}=2\right)$, are determined by the electron-electron interaction and one-electron level spacing $\delta=\varepsilon_{2}-\varepsilon_{1}$. At the moment, we set $C_{1}=C_{2}=1$ [11] and show that this is the general case afterwards. The energy difference, $\Delta=E_{S=0}-E_{S=1}$, is changed by applying a magnetic field $B$ as shown in Fig. 1(a). We disregard the Zeeman splitting of spin states since this is a much smaller energy scale than the orbital effect of the magnetic field in semiconductor heterostructures in use $[5,6]$. The exact condition for this is $E_{\text {Zeeman }} \ll T_{K}$.

The dot is connected to two external leads $L, R$ with free electrons being described by

$$
H_{\text {leads }}=\sum_{\alpha=L, R} \sum_{k \sigma i} \varepsilon_{\alpha k}^{(i)} c_{\alpha, k \sigma}^{(i) \dagger} c_{\alpha, k \sigma}^{(i)} .
$$

The tunneling between the dot and the leads is written as

$$
H_{T}=\sum_{\alpha=L, R} \sum_{k \sigma i}\left(V_{\alpha, i} c_{\alpha, k \sigma}^{(i) \dagger} d_{i \sigma}+\text { H.c. }\right) \text {. }
$$

Here $c_{\alpha, k \sigma}^{(i) \dagger}$ is the creation operator of an electron in lead $\alpha$ with momentum $k$, spin $\sigma$, and orbital symmetry $i$ (= $1,2)$. We assume that the orbital symmetry is conserved in the tunneling processes. Therefore we have two electron "channels" in each lead.

We assume that the state of the dot with $N$ electrons is stable, so that addition/extraction energies, $E^{ \pm} \equiv$ $E(N \pm 1)-E(N) \mp \mu$ where $\mu$ is the Fermi energy in the leads, are positive. We are interested in the case when $E^{ \pm} \gg|\Delta|, \delta$ and also exceed the level broadening $\Gamma_{\alpha}^{i}=$ (a)

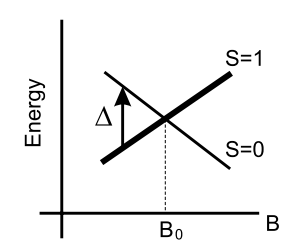

(b)

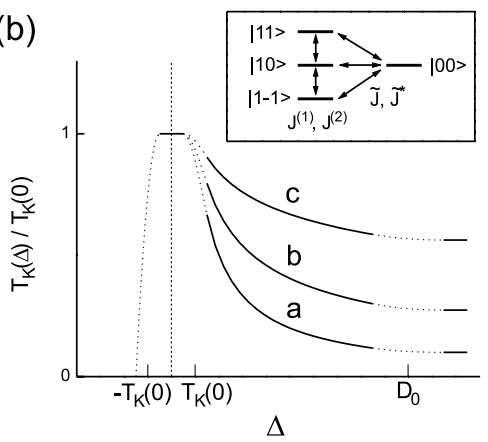

$\Delta$

FIG. 1. (a) The energies of spin-singlet and triplet states in a quantum dot, as functions of magnetic field $B$. The energy difference, $\Delta=E_{S=0}-E_{S=1}$, can be controlled by changing $B$. $\Delta=0$ at $B=B_{0}$, where the transition of the ground state occurs. (b) Schematic drawing of the Kondo temperature, $T_{K}$, as a function of $\Delta$. When $\Delta>0, T_{K}(\Delta) / T_{K}(0)=\left(T_{K}(0) / \Delta\right)^{\gamma}$ where $a, \gamma=1 ; b, 0.5$; and $c, 0.25$ at $T_{K}(0) \ll \Delta \ll D_{0}$ (bandwidth), and $T_{K}(\Delta)$ is a constant at $\Delta \gg D_{0}$. When $\Delta<0$, $T_{K}(\Delta)$ drops to zero suddenly at $|\Delta| \sim T_{K}(0)$. Inset: Spin-flip processes in our model. The exchange couplings $J^{(i)}$ involving spin-triplet states only are accompanied by scattering of conduction electrons of channel $i$. Those involving spin-triplet and singlet states $\left(\tilde{J}, \tilde{J}^{*}\right)$ are accompanied by interchannel scattering of conduction electrons.

$\pi \nu\left|V_{\alpha, i}\right|^{2}$ ( $\nu$ being density of states in the leads) and temperature $T$ (Coulomb blockade region). In this case we can integrate out the states with one or three extra electrons. This is equivalent to Schrieffer-Wolf transformation which is used to obtain the conventional Kondo model [1]. We obtain the following effective low-energy Hamiltonian:

$$
H_{\mathrm{eff}}=H^{S=1}+H^{S=1 \leftrightarrow 0}+H_{\mathrm{eff}}^{\prime}+H_{\mathrm{dot}}
$$

The first term involves components of the spin-triplet state and resembles a conventional Kondo Hamiltonian for $S=1$.

$$
\begin{gathered}
H^{S=1}=\sum_{k k^{\prime}} \sum_{\alpha \beta=L, R} \sum_{i=1,2} J_{\alpha \beta}^{(i)}\left[\hat{S}_{+} c_{\alpha k^{\prime} \downarrow}^{(i) \dagger} c_{\beta k \uparrow}^{(i)}+\hat{S}_{-} c_{\alpha^{\prime} k \uparrow}^{(i) \dagger} c_{\beta k \downarrow}^{(i)}+\hat{S}_{z}\left(c_{\alpha^{\prime} k \uparrow}^{(i) \dagger} c_{\beta k \uparrow}^{(i)}-c_{\alpha^{\prime} k \downarrow}^{(i) \dagger} c_{\beta k \downarrow}^{(i)}\right)\right] \\
=\sum_{k k^{\prime}} \sum_{\alpha \beta=L, R} \sum_{i=1,2} J_{\alpha \beta}^{(i)}\left[\sqrt{2}\left(f_{11}^{\dagger} f_{10}+f_{10}^{\dagger} f_{1-1}\right) c_{\alpha k^{\prime} \downarrow}^{(i) \dagger} c_{\beta k \uparrow}^{(i)}+\sqrt{2}\left(f_{10}^{\dagger} f_{11}+f_{1-1}^{\dagger} f_{10}\right) c_{\alpha k^{\prime} \uparrow}^{(i)} c_{\beta k \downarrow}^{(i)}\right. \\
\left.+\left(f_{11}^{\dagger} f_{11}-f_{1-1}^{\dagger} f_{1-1}\right)\left(c_{\alpha k^{\prime} \uparrow}^{(i) \dagger} c_{\beta k \uparrow}^{(i)}-c_{\alpha k^{\prime} \downarrow}^{(i) \dagger} c_{\beta k \downarrow}^{(i)}\right)\right]
\end{gathered}
$$

Here we have introduced pseudo-fermion operators $f_{S M}^{\dagger}\left(f_{S M}\right)$ which create (annihilate) the state $|S M\rangle$. It is required that $\sum_{S M} f_{S M}^{\dagger} f_{S M}=1$. The second term in $H_{\text {eff }}$ describes the conversion between the spin-triplet and singlet states accompanied by interchannel scattering of conduction electrons

$$
\begin{gathered}
H^{S=1 \leftrightarrow 0}=\sum_{k k^{\prime}} \sum_{\alpha \beta=L, R}\left\{\tilde { J } _ { \alpha \beta } \left[\sqrt{2}\left(f_{11}^{\dagger} f_{00}-f_{00}^{\dagger} f_{1-1}\right) c_{\alpha k^{\prime} \downarrow}^{(1) \dagger} c_{\beta k \uparrow}^{(2)}+\sqrt{2}\left(f_{00}^{\dagger} f_{11}-f_{1-1}^{\dagger} f_{00}\right) c_{\alpha k^{\prime} \uparrow}^{(1)} c_{\beta k \downarrow}^{(2)}\right.\right. \\
\left.\left.-\left(f_{10}^{\dagger} f_{00}+f_{00}^{\dagger} f_{10}\right)\left(c_{\alpha k^{\prime} \uparrow}^{(1) \dagger} c_{\beta k \uparrow}^{(2)}-c_{\alpha k^{\prime} \downarrow}^{(1) \dagger} c_{\beta k \downarrow}^{(2)}\right)\right]+\tilde{J}_{\alpha \beta}^{*}[1 \leftrightarrow 2]\right\} .
\end{gathered}
$$

The third term $H_{\text {eff }}^{\prime}$ represents the scattering processes without change of the dot state and is not relevant for the current discussion. The coupling constants are given by $J_{\alpha \beta}^{(i)}=V_{\alpha, i} V_{\beta, i}^{*} /\left(2 E_{c}\right), \tilde{J}_{\alpha \beta}=V_{\alpha, 1} V_{\beta, 2}^{*} /\left(2 E_{c}\right)$, where $1 / E_{c}=1 / E^{+}+1 / E^{-}$. The Hamiltonian of the dot reads

$$
H_{\mathrm{dot}}=\sum_{S, M} E_{S} f_{S M}^{\dagger} f_{S M} \text {. }
$$

To avoid the complication due to the fact that there are two leads $\alpha=L, R$, we perform a unitary transformation for electron modes in the leads along the lines of Ref. [3]; $\quad c_{k \sigma}^{(i)}=$ $\left(V_{L, i} c_{L, k \sigma}+V_{R, i} c_{R, k \sigma}\right) / V_{i}, \quad \bar{c}_{k \sigma}^{(i)}=\left(V_{R, i}^{*} c_{L, k \sigma}-\right.$ $\left.V_{L, i}^{*} c_{R, k \sigma}\right) / V_{i}$, with $V_{i}=\sqrt{\left|V_{L, i}\right|^{2}+\left|V_{R, i}\right|^{2}}$. The 
modes $\bar{c}_{k \sigma}^{(i)}$ are not coupled to the quantum dot and shall be disregarded. The coupling constants for modes $c_{k \sigma}^{(i)}$ become

$$
\begin{gathered}
J^{(i)}=\frac{\left|V_{L i}\right|^{2}+\left|V_{R i}\right|^{2}}{2 E_{c}}, \\
\tilde{J}=\frac{1}{2 E_{c}} \frac{\left(V_{L 1}^{2}+V_{R 1}^{2}\right)\left(V_{L 2}^{2}+V_{R 2}^{2}\right)}{\sqrt{\left(\left|V_{L 1}\right|^{2}+\left|V_{R 1}\right|^{2}\right)\left(\left|V_{L 2}\right|^{2}+\left|V_{R 2}\right|^{2}\right)}} .
\end{gathered}
$$

The spin-flip processes included in our model are shown in the inset of Fig. 1(b).

We calculate the Kondo temperature $T_{K}$ with the poor man's scaling technique $[12,13]$. By this method, we can properly consider the energies from $T_{K}$ to the upper cutoff. We concentrate on evaluating the exponential part of $T_{K}$. We assume constant density of states in the leads $\nu$ in the energy band of $[-D, D]$. By changing the energy scale from $D$ to $D-|d D|$, we obtain a closed form of the scaling equations for $J^{(1)}, J^{(2)}$, and $\tilde{J}$ in two limits.

In the first limit, the energy difference $|\Delta|$ is negligible $(|\Delta| \ll D)$ and $H_{\text {dot }}$ can be safely disregarded. The scaling equations are best presented in the following matrix form:

$$
\frac{d}{d \ln D}\left(\begin{array}{cc}
J^{(1)} & \tilde{J} \\
\tilde{J}^{*} & J^{(2)}
\end{array}\right)=-2 \nu\left(\begin{array}{cc}
J^{(1)} & \tilde{J} \\
\tilde{J}^{*} & J^{(2)}
\end{array}\right)^{2} .
$$

The equations can be readily rewritten for eigenvalues of the matrix, $J_{ \pm}=\left(J^{(1)}+J^{(2)}\right) / 2 \pm$ $\sqrt{\left(J^{(1)}-J^{(2)}\right)^{2} / 4+|\tilde{J}|^{2}}$. The larger one, $J_{+}$, diverges faster upon decreasing the bandwidth $D$ and hence determines $T_{K}$. If the equations remain valid till the scaling ends $\left(|\Delta| \ll T_{K}\right)$, the Kondo temperature is $T_{K}(0)=$ $D_{0} \exp \left[-1 / 2 \nu J_{+}\right]$. Here $D_{0}$ is the initial bandwidth given by $\sqrt{E^{+} E^{-}}$[14]. In another limiting case, $\Delta \gg D$. In this case the ground state of the dot is spin triplet and the singlet state can be disregarded. $J^{(1)}$ and $J^{(2)}$ evolve independently

$$
\frac{d}{d \ln D} J^{(i)}=-2 \nu J^{(i) 2},
$$

and $\tilde{J}$ does not change. If these equations remain valid in the whole scaling region $\left(\Delta>D_{0}\right)$, it yields $T_{K}(\infty)=$ $D_{0} \exp \left[-1 / 2 \nu J^{(1)}\right]$. Here we assume $J^{(1)} \geq J^{(2)}$. This is the Kondo temperature for spin-triplet localized spins [15].

To determine $T_{K}$ in the intermediate region, $T_{K}(0) \ll$ $\Delta \ll D_{0}$, we match the solutions of Eqs. (13) and (14) at $D \simeq \Delta$. $\tilde{J}$ saturates at this point while $J^{(1)}$ and $J^{(2)}$ continue to grow with decreasing $D$. This yields power law dependence on $\Delta$

$$
T_{K}(\Delta)=T_{K}(0)\left[T_{K}(0) / \Delta\right]^{\gamma},
$$

where $\quad \sqrt{\gamma}=|\tilde{J}| /\left[\sqrt{\left(J^{(1)}-J^{(2)}\right)^{2} / 4+|\tilde{J}|^{2}}+\mid J^{(1)}-\right.$ $\left.J^{(2)} \mid / 2\right]$. The exponent $\gamma$ appears to be nonuniversal, depending on a ratio of the initial coupling constants. In general, $0<\gamma \leq 1$. For $\Delta<0$, all the coupling constants saturate and no Kondo effect is expected, provided $|\Delta| \gg T_{K}(0)$.

In a simple case of the identical couplings, $J^{(1)}=$ $J^{(2)}=\tilde{J}(\equiv J), T_{K}(0)=D_{0} \exp [-1 / 4 \nu J]$. For $\Delta>0$, $T_{K}$ decreases with increasing $\Delta$ as $T_{K}(\Delta)=T_{K}(0)^{2} / \Delta$ $[\gamma=1$ in Eq. (15)] and finally converges to $D_{0} \exp [-1 / 2 \nu J]=T_{K}(0)^{2} / D_{0}$. For $\Delta<0, T_{K}$ drops to zero suddenly at $|\Delta| \sim T_{K}(0)$. The dependence of the Kondo temperature on $\Delta$ is schematically shown in Fig. 1(b).

We have discussed so far the case of $C_{1}=C_{2}=1$ in Eq. (4) for the spin-singlet state. This is not required by symmetry and $C_{1} \neq C_{2}$ in general. To justify the assumption we made, let us consider the renormalization equations for $C_{1} \neq C_{2}$. The coupling constants $\tilde{J}_{1}=C_{1} \tilde{J}$ and $\tilde{J}_{2}=C_{2} \tilde{J}$ are renormalized now in a different way, involving the scattering processes without spin flip in the dot

$$
\begin{array}{r}
H_{\text {eff }}^{\prime}=\sum_{k k^{\prime} \sigma} \sum_{i=1,2}\left[J^{\prime(i)} c_{k^{\prime} \sigma}^{(i) \dagger} c_{k \sigma}^{(i)} \sum_{M} f_{1 M}^{\dagger} f_{1 M}\right. \\
\left.+J^{\prime \prime(i)} c_{k^{\prime} \sigma}^{(i) \dagger} c_{k \sigma}^{(i)} f_{00}^{\dagger} f_{00}\right] .
\end{array}
$$

General scaling equations for $|\Delta| \ll D$ are given by

$$
\begin{gathered}
d \tilde{J}_{1} / d \ln D=-2 \nu\left(J^{(1)}+J^{(2)}\right) \tilde{J}_{1}+\nu J^{\prime} \tilde{J}_{1}, \\
d \tilde{J}_{2} / d \ln D=-2 \nu\left(J^{(1)}+J^{(2)}\right) \tilde{J}_{2}-\nu J^{\prime} \tilde{J}_{2}, \\
d J^{\prime} / d \ln D=8 \nu\left(\left|\tilde{J}_{1}\right|^{2}-\left|\tilde{J}_{2}\right|^{2}\right), \\
d J^{(i)} / d \ln D=-2 \nu\left[J^{(i) 2}+\left(\left|\tilde{J}_{1}\right|^{2}+\left|\tilde{J}_{2}\right|^{2}\right) / 2\right],
\end{gathered}
$$

where $J^{\prime}=J^{\prime(1)}-J^{\prime(2)}-J^{\prime \prime(1)}+J^{\prime \prime(2)}$. When $\Delta \gg D$, the equations are identical to Eq. (14). Our point is that if we concentrate on the most rapidly divergent solutions of Eqs. (17)-(20), which are proportional to $1 / \ln D, \tilde{J}_{1}$ and $\tilde{J}_{2}$ appear to be the same. To this leading order, the renormalization is the same as given by Eq. (13). Consequently the Kondo temperature is the same as that in the case of $C_{1}=C_{2}=1$, apart from a prefactor.

We calculate perturbation corrections to conductance when $T_{K} \ll T \ll E_{c}$. The third order perturbations in $J$ 's yield the logarithmic corrections $G_{K}$ typical for the Kondo effect [1]. At $T \gg|\Delta|$,

$$
\begin{aligned}
G_{K} /\left(2 e^{2} / h\right)= & \sum_{i=1,2} \frac{4 \Gamma_{L}^{i} \Gamma_{R}^{i}}{\left(\Gamma_{L}^{i}+\Gamma_{R}^{i}\right)^{2}} 6 \pi^{2} J^{(i)} \nu\left[\left(J^{(i)} \nu\right)^{2}+|\tilde{J} \nu|^{2}\right] \ln \frac{D_{0}}{T} \\
& +\frac{2\left(\Gamma_{L}^{1} \Gamma_{R}^{2}+\Gamma_{L}^{2} \Gamma_{R}^{1}\right)}{\left(\Gamma_{L}^{1}+\Gamma_{R}^{1}\right)\left(\Gamma_{L}^{2}+\Gamma_{R}^{2}\right)} 12 \pi^{2}\left[J^{(1)} \nu+J^{(2)} \nu\right]|\tilde{J} \nu|^{2} \ln \frac{D_{0}}{T} .
\end{aligned}
$$

The interplay between the spin-singlet and triplet states largely enhances the conductance. In the opposite case, $T \ll|\Delta|$, the interplay becomes less effective and the logarithmic corrections become smaller 


$$
G_{K} /\left(2 e^{2} / h\right)=\sum_{i=1,2} \frac{4 \Gamma_{L}^{i} \Gamma_{R}^{i}}{\left(\Gamma_{L}^{i}+\Gamma_{R}^{i}\right)^{2}} 8 \pi^{2} J^{(i)} \nu\left[\left(J^{(i)} \nu\right)^{2} \ln \frac{D_{0}}{T}+|\tilde{J} \nu|^{2} \ln \frac{D_{0}}{\Delta}\right]
$$

for $\Delta>0$ and disappear for $\Delta<0$. If one varies $\Delta$ at fixed $T$, one sees the enhanced conductance for $|\Delta| \ll T$.

We believe that the conductance approaches the unitary limit $2 e^{2} / h$ at $T \ll T_{K}$ [3] in our model. However, not all the terms in $G_{K}$ can be renormalized to a universal function $G\left(T / T_{K}\right)$. Because of the multichannel nature of our model, one should expect nonuniversal logarithmic terms along with the universal ones. Those, however, will be much smaller than $2 e^{2} / h$.

Recent experiment [9] has shown a significant enhancement of conductance to the values of the order of $e^{2} / h$, around the crossing point between spin-singlet and triplet states, in vertical quantum dots. The conductance remains low in the stability domains of singlet or triplet states. Our results provide a possible theoretical explanation for the results. The conductance increase can be attributed to the Kondo effect enhanced by the competition between the two states, near the crossing point. The Kondo temperature elsewhere is probably low in comparison with the actual temperature, so that no conductance increase is seen.

In conclusion, we have shown that the competition between spin-singlet and triplet states enhances the Kondo effect. This may explain recent experimental observations. We have predicted power law dependence of the Kondo temperature on the energy difference between the states.

The authors are indebted to L.P. Kouwenhoven for suggesting the topic of the research presented, S. De Franceschi, J. M. Elzerman, K. Maijala, S. Sasaki, W. G. van der Wiel, Y. Tokura, L. I. Glazman, M. Pustilnik, and G.E.W. Bauer for valuable discussions. The authors acknowledge financial support from the "Netherlandse Organisatie voor Wetenschappelijk Onderzoek" (NWO). M.E. is also grateful for financial support from the Japan Society for the Promotion of Science for his stay at Delft University of Technology.
[1] J. Kondo, Prog. Theor. Phys. 32, 37 (1964); A. C. Hewson, The Kondo Problem to Heavy Fermions (Cambridge, Cambridge, England, 1993); K. Yosida, Theory of Magnetism (Springer, New York, 1996); D. L. Cox and A. Zawadowski, Adv. Phys. 47, 599 (1998).

[2] D. Goldhaber-Gordon et al., Nature (London) 391, 156 (1998); D. Goldhaber-Gordon et al., Phys. Rev. Lett. 81, 5225 (1998); S. M. Cronenwett et al., Science 281, 540 (1998); F. Simmel et al., Phys. Rev. Lett. 83, 804 (1999).

[3] L. I. Glazman and M. É. Raikh, Pis'ma Zh. Eksp. Teor. Fiz. 47, 378 (1988) [JETP Lett. 47, 452 (1988)].

[4] T. K. Ng and P. A. Lee, Phys. Rev. Lett. 61, 1768 (1988); A. Kawabata, J. Phys. Soc. Jpn. 60, 3222 (1991); S. Hershfield et al., Phys. Rev. Lett. 67, 3720 (1991); Y. Meir et al., Phys. Rev. Lett. 70, 2601 (1993).

[5] S. Tarucha et al., Phys. Rev. Lett. 77, 3613 (1996).

[6] L. P. Kouwenhoven et al., Science 278, 1788 (1997).

[7] T. Inoshita et al., Phys. Rev. B 48, R14725 (1993); T. Pohjola et al., Europhys. Lett. 40, 189 (1997); W. Izumida et al., J. Phys. Soc. Jpn. 67, 2444 (1998); A. Levy Yeyati et al., cond-mat/9901144.

[8] M. Pustilnik et al., Phys. Rev. Lett. 84, 1756 (2000).

[9] S. Sasaki et al., Nature (London) 405, 764 (2000).

[10] Our main results, enhancement of $T_{K}$ and its power law dependence on $\Delta$, are valid also for a simpler model where the levels belong to the same symmetry representation. In this case, however, the level repulsion makes it difficult to organize the degeneracy of spin-singlet and triplet states. This is why we prefer the model in use. Besides, it is experimentally relevant [5].

[11] Considering the matrix element of the Coulomb interaction between $d_{1 \uparrow}^{\dagger} d_{1 \downarrow}^{\dagger}|0\rangle$ and $d_{2 \uparrow}^{\dagger} d_{2 \downarrow}^{\dagger}|0\rangle,\left\langle 22\left|e^{2} / r\right| 11\right\rangle$, one observes that this is indeed the case for $\delta=0$.

[12] P. W. Anderson, J. Phys. C 3, 2436 (1970).

[13] P. Nozières and A. Blandin, J. Phys. (Paris) 41, 193 (1980).

[14] F. D. M. Haldane, J. Phys. C 11, 5015 (1978).

[15] I. Okada and K. Yosida, Prog. Theor. Phys. 49, 1483 (1973). 